\title{
False Heart Rate Feedback and the Perception of Heart Symptoms in Patients with Congenital Heart Disease and Anxiety
}

\author{
Petra A. Karsdorp • Merel Kindt • Simon Rietveld • \\ Walter Everaerd • Barbara J.M. Mulder
}

Published online: 6 January 2009

(C) The Author(s) 2008. This article is published with open access at Springerlink.com

\begin{abstract}
Background Little is known about the mechanisms explaining an increased perception of heart symptoms in congenital heart disease (ConHD). In the present study, it was suggested that a combination of high trait anxiety and disease history increases the perception of heart symptoms. Purpose It was tested whether false heart cues will result in an increased perception of heart symptoms in patients with ConHD and anxiety.

Method Thirty-six patients with ConHD and 44 healthy controls performed two exercise tasks. During one of the exercise tasks, participants were exposed to a false heart cue consisting of false heart rate feedback (regular or irregular). Perceived heart symptoms were assessed and heart rate, arterial partial pressure of $\mathrm{CO}_{2}$, and respirator rate were monitored continuously.

Results In line with the predictions, false heart rate feedback resulted in an increased perception of heart symptoms in high trait anxious patients with ConHD that could not be explained by acute heart dysfunction. However, unexpectedly, this effect was not observed immediately after the false heart rate feedback task but after a second exercise task without false feedback.

Conclusion The results suggest that not the sole presence of ConHD but ConHD in combination with high trait
\end{abstract}

P. A. Karsdorp $\cdot$ M. Kindt $\cdot$ S. Rietveld $\cdot$ W. Everaerd Department of Psychology, University of Amsterdam, Amsterdam, The Netherlands

B. J. Mulder

Department of Cardiology, Academic Medical Center, Amsterdam, The Netherlands

P. A. Karsdorp $(\square)$

Department of Clinical, Medical, and Experimental Psychology,

Maastricht University,

P.O. Box 616, 6200 MD Maastricht, The Netherlands

e-mail: P.Karsdorp@DMKEP.unimaas.nl anxiety results in a vulnerability to overperceive heart symptoms.

Keywords Congenital heart disease $\cdot$ False heart rate feedback · Perceptual bias $\cdot$ Heart symptoms · Trait anxiety

\section{Introduction}

Due to advances in medical and surgical treatment, most of the patients with congenital heart disease (ConHD) will reach adulthood nowadays [1]. Some of these patients still report an increased perception of disturbing heart symptoms $[2,3]$. To develop treatments aimed at diminishing perceived heart symptom, researchers have become interested in the mechanisms that may explain symptom perception.

Several hypotheses have been proposed that may explain these mechanisms. For instance, the influential somatic hypothesis postulates that the heart defect directly and linearly increases perceived heart symptoms (e.g., [4, 5]). However, in sharp contrast to this hypothesis, the severity of heart disease turned out to be only weakly related to physical symptoms (e.g., [2, 5]). The symptom perception hypothesis may explain these findings (e.g., [6]). This hypothesis states that experienced heart symptoms are explained by perceptual biases, such as selective attention and negative interpretation of physical sensations.

High trait anxious patients with chronic disease may be specifically vulnerable to develop perceptual biases [7, 8]. This hypothesis is evolved from an integration of the symptom perception hypothesis [9] and the vulnerabilitystress hypothesis $[10,11]$. That is, disease experiences in combination with long-lasting vulnerabilities, such as high trait anxiety, may result in stressful experiences with disease. These experiences are stored in cognitive memory structures or so-called illness schemes. Perception of 
disease-related cues may activate such an illness scheme, eliciting perceptual biases for disease-related symptoms [6]. As a result, patients with chronic disease may not only perceive physical symptoms due to physiological changes but they may also perceive symptoms based on cues that are unrelated to their actual bodily state, such as diseaserelated environmental cues (e.g., a hospital setting) or false expectations of becoming ill (e.g., [12, 13]).

The aim of the present study was to test whether false feedback of heart rate changes [14] will result in a perceptual bias for heart symptoms in high trait anxious patient with ConHD. We included a healthy control group to test whether a combination of ConHD (disease experience) and trait anxiety influenced the perception of symptoms rather than solely trait anxiety. Each participant accomplished two standardized physical exercise tasks on a treadmill, one with false feedback of either a regular or an irregular heart rate and one without feedback. The regular heart rate feedback was included to test whether awareness of ones heart rate in general or whether specifically an irregular heart rate increased perceived heart symptoms. The exercise tasks were meant to induce ambiguous physiological arousal that may increase uncertainty about the functioning of the heart [15]. An exercise task without feedback was included to exclude the possibility that perceived heart symptoms could be explained solely by the exercise task, by differences in exertion or baseline levels of perceived heart symptoms. To exclude the possibility that the perception of symptoms could be explained by acute heart dysfunction in ConHD, cardiac functioning was monitored continuously. We predicted that the false heart rate feedback, specifically feedback on irregular heart rate, would elicit an increased perception of heart symptoms in high trait anxious patients with ConHD. Moreover, we predicted that this increased perception could not be explained by acute heart dysfunction.

\section{Method}

\section{Participants}

Consecutive adult patients with ConHD were selected from the outpatient clinics of cardiology in the Academic Medical Centre, in Amsterdam. Inclusion criteria were age between 18 and 55 years and no mental retardation. Fortyfour percent $(n=46,21$ women and 25 men, mean $(\mathrm{M})$ age $=$ 31.74 years, standard deviation $(\mathrm{SD})=8.44$, range 18 54 years) of the contacted patients that met the inclusion criteria participated in the experiment. A cardiologist classified the heart defects into complex, moderate, and simple ConHD based on risk of morbidity and mortality, according to the classification system presented at the 32nd
Bethesda Conference [1]. Fifteen participants had a complex ConHD, 16 had a moderate ConHD, and 15 had a simple ConHD. Within the group of patients who met the inclusion criteria, there were no differences between the patients that participated and those who denied participation, with respect to age, gender, and disease severity ( $p>$ $0.05)$. A healthy comparison group $(n=56,28$ women and 28 men, mean age $=28,87$ years, $\mathrm{SD}=9.56$, range 18 54 years) was recruited via advertisements using the inclusion criteria: no acute or chronic diseases, age between 18 and 55 years, and no mental retardation.

Two patients with ConHD showed heart rhythm disturbances during the experiment and therefore had to be excluded from further analysis. Trait anxiety scores of two healthy controls were missing. Two patients with ConHD and two participants from the control group noticed that the regular heart rate feedback was false (see also manipulation check). Six patients with ConHD and eight participants from the control condition noticed that the irregular feedback was false. No differences were found with respect to age, gender, severity of heart disease, trait anxiety, and the perception of heart symptoms during the experiment between the patients that did and did not notice the false heart rate feedback as false. The patients who recognized the heart rate feedback as false were excluded from further analysis, leaving the sample size to 36 patients with ConHD (11 with complex, 11 with moderate, and 14 with simple ConHD) and 44 healthy participants. Twenty-nine of these patients with ConHD had undergone cardiac surgery or intervention to repair or correct their cardiac defect, 17 patients used heart medication, and ten had experienced heart rhythm disturbances in the past. Age and gender did not differ significantly (all $p \mathrm{~s}>0.05$ ) between the final sample of patients with ConHD (17 women and 19 men; mean age $=31.69$ years, $\mathrm{SD}=8.68$, range $19-54$ years) and healthy participants $(18$ women and 27 men; mean age $=$ 29.18 years, $\mathrm{SD}=9.73$, range $18-54$ years).

Due to technical errors, the heart rate, arterial partial pressure of $\mathrm{CO}_{2}$, and respiratory rate were not recorded for one, four, and three patients with ConHD, respectively, and for one healthy control. Therefore, the sample that was included in the analysis of the physiological data was slightly smaller than the sample included for the analysis of the heart symptoms. The local ethical committee of the Amsterdam Medical Centre and the University of Amsterdam approved the study.

\section{Design}

We used a 2 feedback (feedback versus no feedback) $\times 2$ sound (regular versus irregular) fractional factorial design, with feedback as within-subject variable and sound as between-subject variable. The order of the two exercise 
tasks (feedback and no feedback) was randomized among participants. Seventeen patients and 24 healthy controls received the feedback condition first. Moreover, participants were randomly assigned to the regular or the irregular heart sound condition. Sixteen patients and 17 controls were assigned to the regular sound condition.

\section{Materials}

False Heart Rate Feedback The heart rate feedback was created by means of digitally recorded drum sounds, such that it resembled the typical heart sounds that are perceived using a stethoscope. The drum sounds were transformed into two heart sounds of $20 \mathrm{~s}$ : a regular and an irregular heart sound. The regular sound consisted of a heart rate of 115 beats per $\min (\mathrm{b} / \mathrm{min})$. The irregular heart sound consisted of a heart rate of $115 \mathrm{~b} / \mathrm{min}$ followed by skipping heartbeats and a sudden increase in heart rate until $210 \mathrm{~b} / \mathrm{min}$. Two hi-fi speakers placed at the left side of the treadmill were connected to a computer and generated the heart rate sounds.

Trait Anxiety We used a Dutch translation of the 20-item Spielberger trait version of the State-Trait Anxiety Inventory (STAI) to measure trait anxiety [16, 17]. Response categories vary from 1 "almost never" to 4 "almost always". Total scores ranged from 20 to 80. Psychometric performance (reliability and validity) of the trait version of the Dutch STAI is satisfactory [17]. In the present study, the internal reliability (Cronbach's $\alpha$ ) of the STAI was 0.91 .

Perception of Heart Symptoms A three-item symptom scale was constructed that assessed three experienced heart symptoms: heart palpitations, rapid heart rate, and chest pain. Item selection was based on a pilot study that showed that these symptoms are the most frequently experienced symptoms among patients with ConHD $(n=131)$ as compared to healthy controls $(n=111, F \mathrm{~s}(1,240) \geq 6.37$, $\left.p s \leq 0.01, \eta^{2} \mathrm{~s} \geq 0.03\right)$. All participants rated orally on a fivepoint scale the degree to which they experienced these three symptoms ( $1=$ "not at all" to $5=$ "very much"). The heart symptoms were pooled and named "heart symptoms". Total heart symptoms score range from 1 to 5 .

Manipulation Check At the end of the experiment, we assed whether participants were aware of the false heart rate manipulation. The participants rated on a five-point scale how much they believed the false feedback reflected their own heart rate $(1=$ "not at all" to $5=$ "very much"). The mean score of the awareness check among the total sample participating in the experiment was $3.07(\mathrm{SD}=1.41)$. Participants scoring 1 were excluded from the analysis.
Physiology Heart rate, respiratory rate, and arterial partial pressure of $\mathrm{CO}_{2}$ were monitored continuously to exclude the possibility that acute heart dysfunction could explain perceived heart symptoms in ConHD. Heart rate was monitored from three $\mathrm{Ag} / \mathrm{AgCl}$ electrodes, attached via the modified lead- 2 placement. Respiratory rate and arterial partial pressure of $\mathrm{CO}_{2}$ were monitored with a Capnogard etco $_{2}$ monitor (Novametrix, Medical Systems, Walingsford, $\mathrm{CT}$, USA). Arterial partial pressure of $\mathrm{CO}_{2}(\mathrm{mmHg})$ was estimated by measuring the $\mathrm{PetCO}_{2}\left(\mathrm{CO}_{2}\right.$ pressure) in the exhaled air at the end of a normal expiration. A tube was inserted in the nostrils of the participants.

Heart rate was also monitored with a polar vantage heart rate monitor, which was strapped around the chest of the participant (Polar electro Oy, FIN-90440 Kempele, Finland). This monitor signaled the research assistant when the participant's heart rate reached $120 \mathrm{~b} / \mathrm{min}$. At this moment, the false feedback was presented.

\section{Procedure}

The experiment consisted of two exercise tasks on a treadmill for each participant: a feedback and a nonfeedback task. Between the two tasks, there was a 10-min relaxation period. The experiment was conducted under supervision of a cardiologist who sat in an adjacent room and watched the electrocardiogram recording for safety reasons and to determine whether patients showed heart rhythm disturbances or extrasystoles.

Participants were told that the experiment was concerned with the assessment of their physical condition and their experience of physical sensations. After informed consent, the physiological recording equipment was attached to the participant. Moreover, a small microphone was attached near the heart region of the participant. Research assistant A explained that she would register the participants' heart rate by the microphone, the headphone, and the polar heart rate monitor. Both the microphone and the headphone were dummy equipment, used to give the impression that heart sounds were recorded.

The exercise task consisted of six stages in which the speed was raised gradually: $2.5,4,5,6,7$, and $8 \mathrm{~km} / \mathrm{h}$, respectively. Participants had to walk for $30 \mathrm{~s}$ in each stage. It took $10 \mathrm{~s}$ for research assistant B to raise the speed. The exercise task maximally lasted $5 \mathrm{~min}$.

In the feedback task, research assistant A controlled the manipulation of the feedback sounds. As soon as the polar heart rate monitor signaled a heart rate of $120 \mathrm{~b} / \mathrm{min}$, research assistant A said "listen to this!" to assistant B, pressed a button to play one of the false heart rate sounds, and signaled research assistant B to stop raising the speed of the treadmill. In the nonfeedback task, assistant A only signaled to stop raising the speed for $20 \mathrm{~s}$ (normally the 
duration of the heart rate feedback) and pressed a button to mark the physiological signals, which was necessary for the analysis of the physiological data. After the false feedback, or $20 \mathrm{~s}$ in the nonfeedback task, the speed of the treadmill was reset to $2.5 \mathrm{~km} / \mathrm{h}$ for $1 \mathrm{~min}$. During this period, research assistant $\mathrm{B}$ assessed participants' experienced symptoms. After the first exercise task, the physiological recording equipment were disconnected, the participant relaxed for $10 \mathrm{~min}$, and the second exercise task was performed. Participants were interviewed about the heart rate manipulation. Next, all equipment was disconnected and participants were instructed about a questionnaire booklet containing the STAI and biographical questions, which they had to complete at home. Note that the STAI was administered after the exercise tasks in order to avoid that participants would focus too much on their level of anxiety. Finally, the participants were paid and ensured that the false feedback did not reflect their real heart rate. There were no negative reactions after the debriefing. Most participants reacted with surprise after they had been told that the heart rate feedback was false.

\section{Data Reduction}

The dependent variables, that is, perceived heart symptoms after false feedback and after nonfeedback, were $\log ^{10}$ transformed to obtain normal distributions. To test the effect of false heart rate feedback, the dependent variable was derived from change scores, i.e., by subtracting mean perceived heart symptoms in the nonfeedback condition from mean perceived heart symptoms in the feedback condition $\left(\Delta \mathrm{M}_{\text {heart }}=\mathrm{M}_{\text {feedback }}-\mathrm{M}_{\text {nonfeedback }}\right)$. Positive scores of heart symptoms $\left(\Delta \mathrm{M}_{\text {heart }}\right)$ indicate an increased perception of heart symptoms during false feedback relative to nonfeedback.

The physiological data were averaged over a 1-min period, $20 \mathrm{~s}$ after the heart rate had reached $120 \mathrm{~b} / \mathrm{min}$. In this period, perceived symptoms were assessed. For these physiological variables, also change scores were calculated. The sample was split into a low $\left[\mathrm{M}_{\mathrm{STAI}}(\mathrm{SD})=29.86\right.$ (3.50)], medium $\left[\mathrm{M}_{\mathrm{STAI}}(\mathrm{SD})=37.66(2.42)\right]$, and high trait anxiety group $\left[\mathrm{M}_{\mathrm{STAI}}(\mathrm{SD})=50.23(7.22)\right]$ based on tertials of the STAI-trait scores (33rd and 67th percentile were 35 and 41 , respectively).

\section{Statistical Analyses}

To test the prediction that a combination of trait anxiety and ConHD would result in an increased perception of heart symptoms two analyses of variance (ANOVAs) were conducted. Note that we conducted two ANOVAs rather than one overall ANOVA to increase the power of the analysis. Firstly, to test whether feedback order influenced symptom perception, a 2 ConHD (ConHD versus healthy) $\times$ 3 trait anxiety (high, medium versus low) $\times 2$ feedback order (first versus second exercise task with feedback) ANOVA was conducted with change in perceived heart symptoms as the dependent variable and ConHD, trait anxiety, and feedback order as between-subjects factors. Secondly, a 2 ConHD (ConHD versus healthy) $\times 3$ trait anxiety (high, medium versus low) $\times 2$ sound (irregular versus regular) was conducted with change in perceived heart symptoms as the dependent variable and ConHD, trait anxiety, and sound as between-subjects factors to test the prediction that a combination of trait anxiety and ConHD would result in an increased perception of heart symptoms specifically after the irregular heart sound. If interaction effects turned out to be significant, post hoc ANOVAs were performed. Given that we had predictions about the direction of the interaction effects between ConHD, trait anxiety, and sound, one-tailed ANOVAs were used. Note that we also performed the analysis with age, sex, exercise time, and physiology (i.e., change scores of heart rate, $\mathrm{PetCO}_{2}$, or respiratory rate) as covariates. The results of these analyses were not presented here because they yielded very similar results. That is, the significance levels of the main and interaction effects of ConHD, trait anxiety, feedback order, and sound did hardly change and according to Cook's distance, no multivariate outliers were detected.

To test whether acute heart dysfunction could explain differences in perceived heart symptoms, similar ANOVAs were conducted as with heart symptoms but now with change in heart rate, respiratory rate, or $\mathrm{PetCO}_{2}$ as dependent variables. We conducted a Spearman rank correlation and $t$ tests to determine whether either disease severity (healthy controls excluded), use of medication, heart rhythm disturbances in the past, and cardiac interventions (all dichotomous variables) were related to change in perceived heart symptoms. Finally, in order to test whether trait anxiety was influenced by the sole presence of ConHD, a one-way ANOVA was performed with ConHD as between-subjects factor. Moreover, to test whether disease severity influenced trait anxiety levels, a Spearman rank correlation was performed between disease severity and trait anxiety.

\section{Results}

Perception of Heart Symptoms and Feedback Order

The 2 ConHD $\times 3$ trait anxiety $\times 2$ feedback order ANOVA with change in perceived heart symptoms as dependent variable $\left(\Delta \mathrm{M}_{\text {heart }}\right)$ revealed unexpectedly an effect of feedback order. That is, a significant three-way interaction effect was found between ConHD, trait anxiety, and 
feedback order $\left(F(1,68)=3.54, p=0.04, \eta^{2}=0.09\right.$, twotailed; Table 1). Follow-up tests for the two feedback orders separately showed that the predicted two-way interaction between ConHD and trait anxiety was significant $(F(1,35)=$ 2.92, $p=0.03$, one-tailed, $\left.\eta^{2}=0.14\right)$, but only when the participants were exposed to the feedback in the first exercise task $\left(F(1,35)=2.92, p=0.03\right.$, one-tailed, $\eta^{2}=$ $0.14)$. When participants were exposed to the feedback in the second exercise task, no significant findings emerged (all $p \mathrm{~s}>0.05$ ). To further explore the significant two-way interaction in the feedback first condition, differences between patients and healthy controls were tested for the three anxiety groups separately. For the low and medium trait anxious participants, no significant differences emerged between patients with ConHD and healthy controls (all $p \mathrm{~s}>0.05$ ). However, as expected for the high anxious participants, a significant main effect of ConHD was obtained $\left(F(1,11)=19.46, p=0.001, \eta^{2}=0.64\right.$, twotailed). However, unpredictably high anxious patients with ConHD showed a decreased rather than an increased perception of heart symptoms after feedback relative to nonfeedback $\left(\Delta \mathrm{M}_{\text {heart }}\right)$ as compared to healthy controls.

The variable changes in perceived heart symptoms $\left(\Delta \mathrm{M}_{\text {heart }}\right)$ was derived from a subtraction of perceived heart symptoms during nonfeedback $\left(\mathrm{M}_{\text {nonfeedback }}\right)$ from perceived heart symptoms during feedback $\left(\mathrm{M}_{\text {feedback }}\right)$. As a result, it remained unclear why high trait anxious patients with ConHD showed a decreased perception of heart symptoms during feedback relative to nonfeedback $\left(\Delta \mathrm{M}_{\text {heart }}\right)$ as compared to high trait anxious healthy controls in the feedback fist condition. It was both possible that high trait anxious patients with ConHD showed a decreased perception of heart symptoms during false feedback $\left(\mathrm{M}_{\text {feedback }}\right)$ or an increased perception during the nonfeedback condition $\left(\mathrm{M}_{\text {nonfeedback }}\right)$ as compared to high trait anxious healthy controls. Therefore, a post hoc analysis was conducted. A 2 group (ConHD versus healthy) $\times 2$ feedback (feedback versus nonfeedback) repeated measure ANOVA was performed for only the high trait anxious participants in the feedback first condition. The perceived heart symptoms during the false feedback $\left(\mathrm{M}_{\text {feedback }}\right)$ and the nonfeedback condition $\left(\mathrm{M}_{\text {nonfeedback }}\right)$ were the dependent variables with group (ConHD versus healthy) as between-subjects factor and feedback as withinsubject factor. The results showed that high trait anxious patients with ConHD in general perceived more heart symptoms than high trait anxious healthy controls $(F(1$, 14) $=11.00, p=0.01, \eta^{2}=0.44$, two-tailed). Moreover, a significant interaction was found between group and feedback $\left(F(1,14)=5.71, p=0.03, \eta^{2}=0.29\right.$, two-tailed $)$. To explore this interaction further, follow-up analyses were conducted. Firstly, the analysis showed that for the high trait anxious patients with ConHD, heart symptoms during feedback $\left(\mathrm{M}_{\text {feedback }}\right)$ did not differ from heart symptoms during nonfeedback $\left(\mathrm{M}_{\text {nonfeedback }} ; F(1,5)=2.07, p=0.21\right.$, $\eta^{2}=0.29$, two-tailed). For the healthy controls, a trend emerged, that is, they perceived more heart symptoms after feedback $\left(\mathrm{M}_{\text {feedback }}\right)$ than after nonfeedback $\left(\mathrm{M}_{\text {nonfeedback}}\right.$; $F(1,9)=2.07, p=0.08, \eta^{2}=0.31$, two-tailed). Secondly, the analysis showed that, during feedback $\left(\mathrm{M}_{\text {feedback }}\right)$, no group differences emerged $(p>0.05)$. Whereas during nonfeedback, high trait anxious patients with ConHD perceived more heart symptoms than high trait anxious healthy controls $\left(\mathrm{M}_{\text {nonfeedback }} ; F(1,14)=15.46, p<0.01, \eta^{2}=0.53\right.$, two-tailed). This indicated that high trait anxious patients with ConHD perceived more heart symptoms than high trait anxious healthy controls only when they were exposed to false heart rate feedback in a previous exercise task.

Table 1 Means and standard deviations of total heart symptoms in patients with ConHD and controls

\begin{tabular}{|c|c|c|c|c|c|c|c|}
\hline & \multirow[t]{3}{*}{ Feedback } & \multicolumn{3}{|c|}{ Congenital heart disease } & \multicolumn{3}{|l|}{ Control } \\
\hline & & \multirow{2}{*}{$\frac{\text { Low anxiety }^{\mathrm{a}}}{\mathrm{M}(\mathrm{SD})}$} & \multirow{2}{*}{$\frac{\text { Medium anxiety }^{\mathrm{a}}}{\mathrm{M}(\mathrm{SD})}$} & \multirow{2}{*}{$\frac{\text { High anxiety }^{\mathrm{a}}}{\mathrm{M}(\mathrm{SD})}$} & \multirow{2}{*}{$\frac{\text { Low anxiety }^{\mathrm{a}}}{\mathrm{M}(\mathrm{SD})}$} & \multirow{2}{*}{$\frac{\text { Medium anxiety }^{\mathrm{a}}}{\mathrm{M}(\mathrm{SD})}$} & \multirow{2}{*}{$\frac{\text { High anxiety }^{\mathrm{a}}}{\mathrm{M}(\mathrm{SD})}$} \\
\hline & & & & & & & \\
\hline \multicolumn{8}{|l|}{ Feedback order } \\
\hline \multirow[t]{2}{*}{ Feedback first } & Feedback & $1.57(0.53)$ & $1.83(0.43)$ & $1.89(0.58)$ & $1.33(0.33)$ & $1.96(0.63)$ & $1.60(0.21)$ \\
\hline & Nonfeedback & $1.52(0.50)$ & $1.42(0.32)$ & $2.17(0.28)$ & $1.20(0.30)$ & $1.67(0.67)$ & $1.40(0.38)$ \\
\hline \multirow[t]{2}{*}{ Feedback second } & Feedback & $1.72(0.53)$ & $1.43(0.57)$ & $1.56(0.27)$ & $1.48(0.47)$ & $2.11(0.69)$ & $1.33(0.38)$ \\
\hline & Nonfeedback & $1.89(0.54)$ & $1.52(0.47)$ & $1.44(0.27)$ & $1.52(0.47)$ & $1.89(0.88)$ & $1.33(0.47)$ \\
\hline \multicolumn{8}{|l|}{ Sound } \\
\hline \multirow[t]{2}{*}{ Regular sound } & Feedback & $1.76(0.57)$ & $1.54(0.56)$ & $1.53(0.29)$ & $1.33(0.33)$ & $2.07(0.56)$ & $1.50(0.57)$ \\
\hline & Nonfeedback & $1.86(0.50)$ & $1.50(0.44)$ & $1.60(0.37)$ & $1.30(0.31)$ & $1.83(0.74)$ & $1.21(0.17)$ \\
\hline \multirow[t]{2}{*}{ Irregular sound } & Feedback & $1.50(0.46)$ & $1.67(0.58)$ & $1.86(0.54)$ & $1.67(0.58)$ & $2.00(0.78)$ & $1.56(0.27)$ \\
\hline & Nonfeedback & $1.50(0.55)$ & $1.44(0.38)$ & $1.95(0.49)$ & $1.67(0.67)$ & $1.71(0.84)$ & $1.61(0.49)$ \\
\hline
\end{tabular}

Feedback first $=$ feedback during the first exercise task. Feedback second $=$ feedback during the second exercise task

$M$ mean, $S D$ standard deviation

${ }^{\text {a }}$ Based on tertials of the STAI trait, the sample was split into a low, medium, and high anxious group 
Perception of Heart Symptoms and Feedback Sound

The 2 ConHD $\times 3$ trait anxiety $\times 2$ sound ANOVA with change in perceived heart symptoms as dependent variable $\left(\Delta \mathrm{M}_{\text {heart }}\right)$ did not reveal a significant effect of sound (all $p s>0.05$; Table 1). Moreover, no other significant main or interaction effects emerged between ConHD and trait anxiety (all $p \mathrm{~s}>0.05$ ).

\section{Heart Function}

The 2 ConHD $\times 3$ trait anxiety $\times 2$ feedback order ANOVA with change in heart rate as dependent variable only showed a significant two-way interaction between group and feedback order $\left(F(1,66)=5.67, p=0.02, \eta^{2}=0.08\right)$. Follow-up tests for the two feedback orders separately did not reveal significant group differences in heart rate changes $(p>0.05)$. Analyses of $\mathrm{PetCO}_{2}$ or respiratory rate as dependent variables did not produce significant main or interaction effects (all $p s>0.05$ ).

The 2 ConHD $\times 3$ trait anxiety $\times 2$ sound ANOVA with heart rate as dependent variable showed a larger increase in heart rate after the regular sound than after the irregular sound in all participants $\left(F(1,66)=4.53, p=0.04, \eta^{2}=\right.$ 0.06). For $\mathrm{PetCO}_{2}$ as the dependent variable, a significant two-way interaction emerged between trait anxiety and sound $\left(F(1,63)=4.87, p=0.01, \eta^{2}=0.13\right)$. Follow-up tests for the anxiety groups separately revealed a larger increase in $\mathrm{PetCO}_{2}$ after the regular sound than after the irregular sound for only the medium anxious participants $(F(1,23)=$ 9.37, $\left.p=0.01, \eta^{2}=0.29\right)$. For respiratory rate as the dependent variable, no significant main or interaction effects emerged (all $p \mathrm{~s}>0.05$ ).

Severity of heart disease was unrelated to change in reported heart symptoms ( $r(36)=0.05, p=0.77)$. Moreover, the same pattern was observed for use of medication, heart rhythm disturbances in the past, and cardiac interventions (all $p \mathrm{~s}>0.05$ ).

\section{Trait Anxiety}

A one-way ANOVA showed that patients with ConHD $(\mathrm{M}=40.33, \mathrm{SD}=9.59)$ did not significantly differ on trait anxiety from controls $(\mathrm{M}=38.06, \mathrm{SD}=9.44, F(1,78)=$ 1.13, $\left.p=0.29, \eta^{2}=0.01\right)$. Severity of heart disease was unrelated to trait anxiety $(r(36)=-0.10, p=0.55)$.

\section{Discussion}

The prediction that false heart rate feedback would increase the perception of heart symptoms in individuals with ConHD who are also high trait anxious was only partially confirmed. As predicted, high trait anxious patients with ConHD showed an increased perception of heart symptoms subsequently to the false heart rate feedback. However, unpredictably, this increased symptom perception was not yielded immediately after the false heart rate feedback, but only in the second exercise task subsequent to the task with false heart rate feedback. Unexpectedly, the irregular feedback did not induce stronger perceptual biases than the regular feedback. As predicted, the increased perception of heart symptoms in high trait anxious patients with ConHD could not be explained by simultaneous cardiac dysfunction. Patients with ConHD and healthy controls did not differ in their physiological responses. Moreover, the patients with ConHD included in this study did not show uncommon heart rhythm disturbances or extrasystoles during the experiment. Finally, severity of heart disease was unrelated to the perception of heart symptoms. In contrast to the somatic hypothesis, the present study suggests that the sole presence of cardiac dysfunction does not necessarily result in the perception of heart symptoms. Instead, exposure to false heart cues and perceptual biases, due to a combination of trait anxiety and a history of ConHD, seems to moderate the relation between heart function and perceived heart symptoms in ConHD.

There are several possible explanations for the finding that the false heart rate feedback did not immediately evoke biased perception in high trait anxious patients with ConHD. Firstly, it is possible that high trait anxious patients with ConHD demonstrated an increased perception of heart symptoms in general that was not influenced by the false heart rate feedback due to a ceiling effect. In line with this suggestion is the finding that high trait anxious patients with ConHD who received the false feedback in the first exercise task perceived more heart symptoms than high trait anxious healthy controls. Moreover, the high trait anxious patients with ConHD did not show any significant changes in symptom perception as compared to the nonfeedback condition. On the other hand, it is unlikely that a ceiling effect in high trait anxious patients with ConHD explained all findings, since high trait anxious patients with ConHD who received the false feedback in the second exercise task did not perceive more heart symptoms than high trait anxious healthy controls.

An alternative explanation for the current results may be provided by the competition of cues hypothesis [6]. According to this hypothesis, individuals attend less to internal sensory information in the presence of salient external environmental information. Possibly, high anxious patients may have allocated their attention to the false heart rate feedback (e.g., [18-20]), rather than to heart rate changes in their own body. This may have counteracted the tendency of high trait anxious patients with ConHD to perceive more heart-related symptoms immediately after 
the false heart rate feedback as compared to high trait anxious healthy controls. But during the second exercise, task high trait anxious patients with ConHD scanned their body for possible signs of cardiac dysfunction and misinterpreted harmless heart rate changes as signs of cardiac dysfunction [6]. The finding that specifically high trait anxious patients with ConHD demonstrated perceptual biases for heart symptoms after exposure to heart-related cues is in accordance with previous research. Previous research has demonstrated that acute stress [8] and perceived heart rate changes [7] elicit perceptual biases in specifically high trait anxious patients with ConHD. This indicates that the combination of trait anxiety and a history of ConHD may induce overperception of heart symptoms after exposure to heart-related cues.

There are several explanations for the finding that the regular heart rate feedback evoked a similar increase in perceived symptoms as the irregular heart rate feedback in high trait anxious patients with ConHD. Firstly, the increased perception of heart symptoms after the regular and irregular feedback may be due to the fact that the regular and irregular heart rate feedback were both false and incongruent to the real heart rate. Alternatively, the increased perception of heart symptoms may be evoked by feedback of an increased heart rate, irrespective of whether it is regular or not, or by any cue that is somehow associated with the heart. Finally, the communication between the research assistants about the heart rate of the participant before the regular and irregular heart rate feedback may have caused the increased perception of heart symptoms.

An alternative explanation for the increased perception of heart symptoms in specifically high anxious patients with ConHD is that this feedback induced parallel changes in the viscera and autonomic nervous system (e.g., [21, 22]). The present study showed that the regular heart rate feedback relative to the irregular heart rate feedback tended to induced larger increases in heart rate immediately after exposure to the false feedback in all participants and increases in arterial partial pressure of $\mathrm{CO}_{2}$ in medium anxious participants. However, high anxious patients with ConHD did not show a different physiological response during the assessment of heart symptoms than high trait anxious healthy individuals. Moreover, the effect of the regular feedback on symptom perception did not differ from the effect of the irregular feedback. Therefore, physiological responses induced by the false feedback could not explain the observed increase in symptom perception in this sample.

We assumed that the level of trait anxiety is independent of the presence of heart disease. Alternatively, the heart defect may have increased the level of trait anxiety [23] or trait anxiety may have increased the severity of heart disease [24]. Little support for these assumptions has been found in the present study, since trait anxiety was unrelated to the severity of heart disease.

Some caution is warranted about the generalization of the findings, as the response rate and sample sizes were relatively low. Since the order of the exercise tasks with and without feedback unexpectedly affected the perception of heart symptoms, it is possible that the power was too small to reveal significant effects of the regular or the irregular heart rate feedback. Another limitation of the present study is that experiences with disease in general rather than with heart disease are related to an increased perception of heart symptoms, as we only included a healthy control group. Conversely, it has been shown that patients with asthma display biased perceptions towards asthma-related symptoms (breathlessness) and not towards heart-related symptoms such as heart pounding [25]. This may imply that an increased perception of heart-related symptoms is specific to patients with ConHD. An additional limitation of the present study is that the STAI questionnaire was administered after study participation. It is possible that, even though participants completed the questionnaire at home, exposure to the false heart rate feedback influenced the report of trait anxiety afterwards. However, it is unlikely that the procedure itself influenced our findings because in another similar study on ConHD, the STAI was completed before study participation [8]. Since this study obtained similar results as the present study, in that specifically high trait anxious patients with ConHD showed perceptual biases for heart sensations, it seems unlikely that the procedure has determined the results of the present study. A final limitation of the present study is that symptom perception is assessed in a controlled laboratory setting rather than in real life situations. It is possible that the false heart rate feedback did not induce as much stress as heartrelated stimuli would have in daily life [26]. The fact that a cardiologist was present during the experiment may have reduced the level of stress even more. Therefore, it is possible that the present findings underestimate biased symptom perception in high trait anxious patients with ConHD in real life.

The present findings suggest that ConHD in combination with high trait anxiety may explain overperception of heart symptoms when they are exposed to heart-related stimuli in the past. In case of acute heart dysfunction, this may be an adaptive response because patients may act appropriately and in turn increase their survival chances. However, in the absence of acute heart dysfunction, overperception of heart symptoms may unduly result in avoidance of physical and social activities [19] and unnecessary doctor visits (e.g., [27]). Psychological interventions may be developed to diminish possible detrimental perceptual biases. Psychological interventions such as biofeedback training [28] or 
interpretive bias training [29] may be beneficial, because it increases the accuracy of heart beat perception and may reduce the negative effect of false believes and expectations about heart function.

Acknowledgements We gratefully acknowledge Kiki Hohnen for her grammatical advice and Sachlan Apil, Sophie Zeyl, Tim Ziermans, Beate Lauxterman, Greetje Carlier, and Simone van Geel for their assistance in data collection. The report was written as part of a project funded by the Dutch Heart Foundation (no. 99.038).

Open Access This article is distributed under the terms of the Creative Commons Attribution Noncommercial License which permits any noncommercial use, distribution, and reproduction in any medium, provided the original author(s) and source are credited.

\section{References}

1. Warnes CA, Liberthson R, Danielson G, Dore A, Harris L, Hoffman JIE, Sommerville J, Williams RG, Webb GD. Task force 1: the changing profile of congenital heart disease in adult life. $\mathrm{J}$ Am Coll Cardiol. 2001;37:1170-5.

2. Kamphuis M, Ottenkamp J, Vliegen HW, Vogels T, Zwinderman KH, Kamphuis RP, Verloove-Vanhorick SP. Health related quality of life and health status in adult survivors with previously operated complex congenital heart disease. Heart. 2002;87:356-62.

3. Rietveld S, Mulder BJ, Van Beest I, Lubbers W, Prins PJ, Vioen S, Bennebroek-Evererz F, Vos A, Casteelen G, Karsdorp P. Negative thoughts in adults with congenital heart disease. Int $\mathrm{J}$ Cardiol 2002;86:19-26.

4. Hager A, Hess J. Comparison of health related quality of life with cardiopulmonary exercise testing in adolescents and adults with congenital heart disease. Heart 2005;91:517-20.

5. Rose M, Köhler K, Köhler F, Sawitzky B, Fliege H, Klap BF. Determinants of the quality of life of patients with congenital heart disease. Qual Life Res. 2005;14:35-43.

6. Pennebaker JW. The psychology of physical symptoms. New York: Springer; 1982.

7. Karsdorp PA, Kindt M, Everaerd W, Mulder BJM. Preattentive processing of heart cues and the perception of heart symptoms in congenital heart disease. Behav Res Ther. 2007;45:1893-902.

8. Karsdorp PA, Kindt M, Rietveld S, Everaerd W, Mulder BJM. Stress-induced heart symptoms and perceptual biases in patients with congenital heart disease. Int J Cardiol. 2007;114:352-7.

9. Watson D, Pennebaker JW. Health complaints, stress and distress: exploring the central role of negative affectivity. Psychol Rev 1989;96:234-54.

10. De Beurs E, Comijs H, Twisk JWR, Sonnenberg C, Beekman ATF, Deeg D. Stability and change of emotional functioning in late life: modelling of vulnerability profiles. J Affect Disord. 2005;84:53-62.

11. Zvolensky MJ, Kotov R, Antipova AV, Schmidt NB. Diathesis stress model for panic-related distress: a test in a Russian epidemiological sample. Behav Res Ther. 2005;43:521-32.

12. Hoehn-Saric R, McLeod DR. Anxiety and arousal: physiological changes and their perception. J Affect Disord. 2000;61:217-24.

13. Koyama T, McHaffie JG, Laurienti PJ, Coghill RC. The subjective experience of pain: where expectations become reality. Proc Natl Acad Sci. 2005;102:12950-5.

14. Vallins S. Cognitive effects of false heart-rate feedback. J Pers Soc Psychol. 1966;4:400-8.

15. Schachter S, Singer J. Cognitive, social, and physiological determinants of emotional state. Psychol Rev. 1962;69:121-8.

16. Spielberger CD, Gorsuch R, Lusheve R. STAI manual for the State-Trait Anxiety Inventory. Palo Alto, CA: Consulting Psychologists; 1970.

17. Van der Ploeg HM, Defares HB, Spielberger CB. Handleiding bij de zelfbeoordelingsvragenlijst. Een nederlandse bewerking van de Spielberger State-Trait Anxiety Inventory. [Validity of the ZelfBeoordelings-Vragenlijst. A Dutch version of the Spielberger State-Trait Anxiety Inventory]. Lisse: Zwets and Zeitlinger; 1979.

18. Parkinson B. Emotional effects of false autonomic feedback. Psychol Bull. 1985;98:471-94.

19. Rietveld S, Karsdorp PA, Mulder BJM. Heartbeat sensitivity in adults with congenital heart disease. Int J Behav Med 2004;11: 203-11.

20. Stern RM, Botto RW, Herrick CD. Behavioral and physiological effects of false heart rate feedback: a replication and extension. Psychophysiology. 1972;9:21-9.

21. Crucian GP, Hughes JD, Barrett AM, Williamson DJG, Bauer RM, Bowers D, Heilam KM. Emotional and physiological responses to false feedback. Cortex 2000;36:623-47.

22. Ehlers A, Margraf J, Roth WT, Taylor B, Birbaumer N. Anxiety induced by false heart rate feedback in patients with panic disorder. Behav Res Ther. 1988;26:1-11.

23. Connoly D, McClowry S, Hayman L, Mahony L, Artman M. Posttraumatic stress disorder in children after cardiac surgery. J Pediatr. 2004;144:480-4.

24. Sher L. Type D personality: the heart, stress, and cortisol. Q J Med. 2005;98:232-9.

25. Rietveld S, Houtveen JH. Acquired sensitivity to relevant physiological activity in patients with chronic health problems. Behav Res Ther. 2004;42:137-53.

26. Rozanski A, Blumenthal JA, Kaplan J. Impact of psychological factors on the pathogenesis of cardiovascular disease and implications for therapy. Circulation. 1999;99:2192-217.

27. Ehlers A, Mayou RA, Sprigings DC, Birkhead J. Psychological and perceptual factors associated with arrhythmias and benign palpitations. Psychosom Med. 2000;62:693-702.

28. O'Brien WH, Reid GJ, Jones KR. Differences in heartbeat awareness among males with higher and lower levels of systolic blood pressure. Int J Psychophysiol 1998;29:53-63.

29. Salemink E, van den Hout M, Kindt M. Trained interpretive bias: validity and effects on anxiety. J Behav Ther Exp Psychiatry. 2007;38:212-24. 